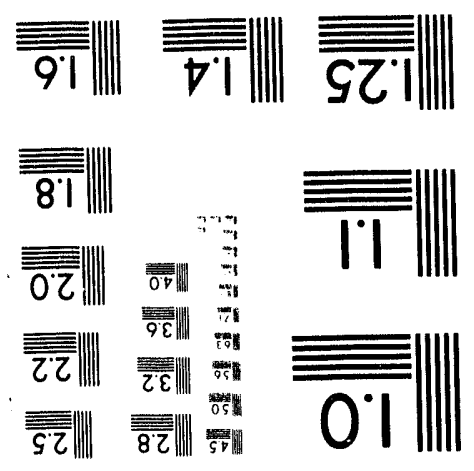



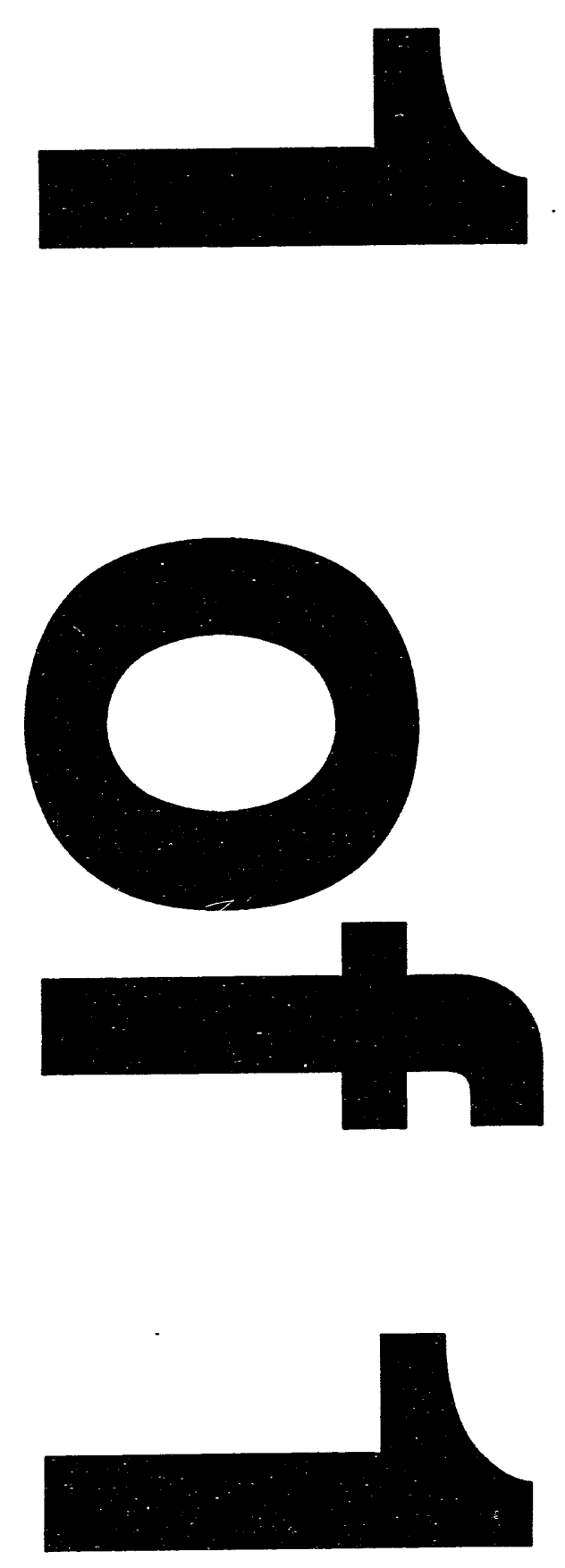
ORNL/TM-12669

\section{USING THE LOG-NORMAL DISTRIBUTION IN ANALYZING AEROSOLS: THE MATHEMATICAL REASONING UNDERLYING THE VARIOUS DIAMETERS USED AND THE PLOTTING PROCEDURE}

Melvin L. Tobias

Date: December 1993

\footnotetext{
Prepared by

OAK RIDGE NATIONAL LABORATORY

Oak Ridge, Tennessee 37831 managed by

MARTIN MARIETTA ENERGY SYSTEMS, INC. for the

U.S. DEPARTMENT OF ENERGY under Contract No. DE-AC05-84OR21400
} 


\title{
USING THE LOG-NORMAL DISTRIBUTION IN ANALYZING AEROSOLS: THE MATHEMATICAL REASONING \\ UNDERLYING THE VARIOUS DIAMETERS USED AND THE PLOTTING PROCEDURE
}

\author{
Melvin L. Tobias
}

\begin{abstract}
This paper is intended to clarify the use of terminology and notation used in the analysis of aerosol particle sizing on the basis of the log-normal distribution. The connection between the log-normal distribution and the normal distribution is shown; the mathematical basis for the various diameters used in the literature is developed, and the reasoning behind the graphical presentation on so-called "log-normal" graph paper is presented.
\end{abstract}




\section{INTRODUCTION}

The purpose of this note is to clarify certain terminology used in the analysis and discussion of fitting aerosol size data into the customarily used log-normal distribution. Specifically, we shall first briefly indicate the relation of the log-normal distribution to the normal distribution. Next, the method of graphical correlation will be described. Finally, we shall discuss and derive the quantities listed on pp. 113-114 of the Handbook On Aerosols (Ref.1) and give them a precise definition. These quantities are the various diameters discussed in aerosol technology that are generated from the mathematics of the log-normal distribution. We shall attempt to give them precise and understandable definitions. The important fact that, in contrast to the normal distribution, the moments of a log-normal distribution remain in a log-normal distribution will be explained. The great simplification which results will be used to obtain the desired size definitions quickly and without tedious manipulation.

The derivations and information presented here are not claimed to be original and mathematical rigor has been sacrificed for the sake of brevity. (It is hoped that the presentation is not too flawed by this shortcut.) The justification for this presentation is that, in this writer's opinion, expositions in the literature are scattered, mathematical notations can be obscure, some matters are glossed over, and, worst of all, the nomenclature is often ambiguous. The result is that many readers are confused or misled, myself among them. It is hoped that a consistent and brief presentation will be worth while. 


\section{THE LOG-NORMAL DISTRIBUTION AND THE NORMAL DISTRIBUTION}

It is customary to treat the size distribution of aerosols on the basis of the so-called log-normal distribution function. "Log-normal" is a shorthand term for taking a normal distribution in the legarithm of the particle radius rather than the radius itself. The formulation is:

$$
N(\ln r) d(\ln r)=e^{\frac{-\left(\ln r-c_{1}\right)^{2}}{c_{2}}} d(\ln r)
$$

Here " $r$ " is the radius of a particle, "In" refers to the natural logarithm, $C_{1}$ and $C_{2}$ are constants (which will be discussed later) and $N(\ln r) d(\ln r)$ is the number of particles which lie between $\ln r$ and $\ln r+d(\ln r) . N(\ln r)$ and the corresponding term on the righthand side denote the distribution or probability density function. We repeat that this is a distribution in the logarithm of $r$, not $r$ itself.

Now this distribution is clearly based upon the concept of the normal distribution of enror law. If values of $x$ are "normally distributed," we say that the probability that a quantity $x$ lies between $x$ and $x+d x$ is $P(x) d x$, where:

$$
P(x) d x=\frac{1}{\sqrt{2 \pi \sigma^{2}}} e^{-\frac{(x-\mu)^{2}}{2 \sigma^{2}}} d x
$$

This is the "normal density function with mean $\mu$ and standard deviation $\sigma$ (Ref. 2). To explain this further, first integrate formula (2) from $-\infty$ to $+\infty$ :

$$
I=\int_{-\infty}^{-} P(x) d x=\frac{1}{\sigma \sqrt{2 \pi}} \int_{-\infty}^{\infty} e^{-\frac{(x-\mu)^{2}}{2 \sigma^{2}}} d x
$$

Make the substitution $w=(x-\mu) /(\sqrt{2} \sigma)$. Then

$$
I=\frac{1}{\sigma \sqrt{2 \pi}} \int_{-\infty}^{\infty} e^{-w^{2}} \sqrt{2} \sigma d w=\frac{1}{\sqrt{\pi}} \int_{-\infty}^{\infty} e^{-w^{2}} d w .
$$


The integral

$$
\int_{-\infty}^{\infty} e^{-w^{2}} d w=2 \int_{0}^{\infty} e^{-w^{2}} d w=\sqrt{\pi}
$$

as can be seen from any table of definite integrals such as Ref. 3. The value of the integral I is 1 , which is what we expect of a probability density function integrated over all possible values.

Next we show that $\mu$ is indeed the mean value of $x$ over the normal distribution function. The mean value of a quantity weighted by a distribution function $f(x)$ of a range from $x_{1}$ to $x_{2}$ is defined as

$$
x_{2 v}=\frac{\int_{x_{1}}^{x_{2}} x f(x) d x}{\int_{x_{1}}^{x_{2}} f(x) d x}
$$

The denominator is the normalizing factor.

Taking $f(x)$ as the $P(x)$ of Eq. (2) and assuming we can extend the range of integration from $-\infty$ to $+\infty$, the mean value of $x$ for 'he normal distribution is:

$$
x_{a v}=\frac{1}{\sigma \sqrt{2 \pi}} \int_{-\infty}^{\infty} x e^{-\frac{(x-\mu)^{2}}{2 \sigma^{2}}} d x
$$

Setting $w=(x-\mu) /(\sqrt{ } 2 \sigma)$ as before, we have

$$
\begin{aligned}
x_{a v} & =\frac{1}{\sigma \sqrt{2 \pi}} \int_{-\infty}^{\infty}(\sigma \sqrt{2} w+\mu) e^{-w^{2}} \sigma \sqrt{2} d w \\
& =\frac{1}{\sqrt{\pi}}\left[\int_{-\infty}^{-} \sqrt{2} \sigma w e^{-w^{2}} d w+\mu \int_{-\infty}^{\infty} e^{-w^{2}} d w\right] .
\end{aligned}
$$

The first of the two integrals is obviously zero because of its odd-function argument. As shown in Eqs. (3)-(5), the second integral is $\sqrt{\pi}$. We see then that $x_{\mathrm{av}}=\mu$, so that $\mu$ is equal to the mean value of $x$. 

$\left(\mathrm{x}^{2}\right)_{\mathrm{av}}$ :

Next, we show that $\sigma$ is the standard deviation by obtaining the mean value of $x^{2}$,

$$
\left(x^{2}\right)_{a v}=\frac{1}{\sigma \sqrt{2 \pi}} \int_{-\infty}^{\infty} x^{2} e^{-\frac{(x-\mu)^{2}}{2 \sigma^{2}}} d x
$$

Again making the substitution $w=(x-\mu) /(\sqrt{2} \sigma)$ leads to

$$
\begin{aligned}
x_{a v} a v & =\frac{1}{\sigma \sqrt{2 \pi}} \int_{-\infty}^{\infty}(\sigma \sqrt{2} w+\mu)^{2} e^{-w^{2}} \sigma \sqrt{2} d w \\
& =\frac{1}{\sqrt{\pi}}\left[\int_{-\infty}^{\infty}\left(2 w^{2} \sigma^{2}+2 \sqrt{2} w \sigma \mu+\mu^{2}\right) e^{-w^{2}} d w\right] .
\end{aligned}
$$

Since the middle term of the argument of the integral is an odd function, we have

$$
\left(\mathrm{x}^{2}\right)_{\mathrm{av}}=\frac{1}{\sqrt{\pi}}\left[2 \sigma^{2} \int_{-\infty}^{-} \mathrm{w}^{2} \mathrm{e}^{-w^{2}} \mathrm{dw}+\mu^{2} \sqrt{\pi}\right]
$$

and since (Ref. 3, formula 494),

$$
\begin{gathered}
\int_{-\infty}^{\infty} w^{2} e^{-w^{2}} d w=\frac{\sqrt{\pi}}{2} \\
\left(x^{2}\right)_{a v}=\sigma^{2}+\mu^{2}=\sigma^{2}+\left(x_{a v}\right)^{2} .
\end{gathered}
$$

This is the same relationship as that which exists between the "mean of the squares" $\left(\mathrm{x}^{2}\right)_{\mathrm{av}}$, the square of the mean, $\left(\mathrm{x}_{\mathrm{av}}\right)^{2}$, and the square of the standard deviation for a finite collection of $\mathrm{N}$ numbers $\mathrm{x}_{\mathbf{i}}$. There, we define

$$
x_{\mathrm{av}} \equiv \frac{\sum_{i=1}^{N} x_{i}}{N} ;\left(x^{2}\right)_{A v} \equiv \frac{\sum_{i=1}^{N} x_{i}^{2}}{N} ; \sigma^{2}=\frac{\sum_{i=1}^{N}\left(x_{i}-x_{a v}\right)^{2}}{N}
$$

Expanding the square term in the definition for $\sigma^{2}$,

$$
\sigma^{2}=\left(\mathrm{x}^{2}\right)_{\mathrm{av}}-2\left(\mathrm{x}_{\mathrm{av}}\right)^{2}+\left(\mathrm{x}_{\mathrm{av}}\right)^{2}=\left(\mathrm{x}^{2}\right)_{\mathrm{av}}-\left(\mathrm{x}_{\mathrm{av}}\right)^{2}
$$




\section{GRAPHICAL REPRESENTATION}

If a population distribution is normal or log-normal, it is possible to represent it as a straight line on paper that is specially designed for the purpose. This technique was invented by Hatch (Ref. 4). As most textbooks either gloss over this method, or explain it in an abstruse way, the following simple exposition is offered.

Suppose the distribution is of the form:

$$
M(x)=\frac{1}{\sigma \sqrt{2 \pi}} \int_{-\infty}^{x} e^{-\frac{\left(1-x_{a v}\right)^{2}}{2 \sigma^{2}}} d t,
$$

where $M(x)$ is the fraction of the population number that have values ranging from $-\infty$ to $x$. As before, the integration variable on the right hand side is changed by the substitution

$$
w=\frac{t-x_{2 v}}{\sigma \sqrt{2}}
$$

Then, $d t=(\sigma \sqrt{2}) d w$, and the upper integration limit becomes $\left(x-x_{a v}\right) /(\sigma \sqrt{2})$. We then have:

$$
M(x)=\frac{1}{\sqrt{\pi}} \int_{-\infty}^{\frac{x-x_{m}}{\sigma \sqrt{2}}} e^{-w^{2}} d w
$$

Now $x$ is either greater, equal to, or less than $x_{a v}$. We then have, for $x<x_{a v}$

$$
M(x)=\frac{1}{\sqrt{\pi}} \int_{-\infty}^{\frac{-\left(x_{w}-x\right)}{\sigma \sqrt{2}}} e^{-w^{2}} d w=\frac{1}{\sqrt{\pi}} \int_{\frac{x_{w}-x}{\sigma \sqrt{2}}}^{\infty} e^{-w^{2}} d w
$$

or

$$
M(x)=\frac{1}{2}-\frac{1}{2} \operatorname{erf}\left(\frac{x_{\mathrm{av}}-x}{\sigma \sqrt{2}}\right),
$$


where the "erf" function is defined as

$$
\operatorname{erf}(y)=\frac{2}{\sqrt{\pi}} \int_{0}^{y} e^{-t^{2}} d t
$$

[This function is tabulated in Ref. (3) and also obtainable from packaged computer subroutines.] Note that $\operatorname{erf}(-y)=-\operatorname{erf}(y)$.

To further the discussion we propose inventing an inverse function erf ${ }^{-1}(w)$. It is to be defined as

$$
w=\frac{2}{\sqrt{\pi}} \int_{0}^{e f^{-1}(w)} e^{-t^{2}} d t
$$

In words, $\operatorname{erf}^{-1}(w)$ is that number whose erf is $w$. (This is analogous to the inverse trigonmetric functions, where $\tan ^{-1} w$ is the number value of an angle in radius whose tangent is W.)

Rearranging Eq. (18) as

$$
\operatorname{erf}\left[\left(x_{\mathrm{av}}-\mathrm{x}\right) /(\sigma \sqrt{2})\right]=1-2 \mathrm{M}(\mathrm{x})
$$

which can be changed to

$$
\operatorname{erf}\left[\left(x-x_{a v}\right) /(\sigma \sqrt{2})\right]=2 M(x)-1
$$

we can write

$$
\frac{\left(x-x_{\text {av }}\right)}{\sigma \sqrt{2}}=\operatorname{erf}^{-1}[2 M(x)-1]
$$

or

$$
x=x_{\mathrm{av}}+\sigma \sqrt{2}\left\{\operatorname{erf}^{-1}[2 M(x)-1]\right\}
$$

The quantity $x$ is therefore linear in the function $\operatorname{erf}^{-1}[2 M(x)-1]$. Now if $x<x_{a v}$, $M(x)$, the cumulative fraction of quantities that have values up to $x$, will range between 0 and 0.5 . If $M(x)$ is 0 then $\operatorname{erf}^{-1}[2 M(x)-1]=-\infty$, and as $x$ increases up to $x_{a v}, M(x)$ will 
increase to 0.5 , for which $\operatorname{erf}^{-1}[-1+2 *(1 / 2)]=0$. We can lay off distances on a piece of paper along a straight line to the left of an origin that are in proportion to the value of $x$ that would give a value of $[2 M(x)-1]$. That is, two distances $d_{1}, d_{2}$ on the paper that correspond to a pair of values $x_{1}, x_{2}$ are in the ratio $d_{1} / d_{2}=\left\{\operatorname{erf}^{-1}\left[2 M\left(x_{1}\right)-1\right]\right\} /\left\{\operatorname{erf}^{-1}[2\right.$ $\left.\left.M\left(x_{2}\right)-1\right]\right\}$. Note that if $M(x)=0.5$, the distance would be zero, and if $M(x)$ were 0.0 , the distance would be $-\infty$.

Suppose now that $x>x_{a v}$. We now have

$$
M(x)=\frac{1}{\sqrt{\pi}} \int_{-\infty}^{\frac{x-x_{w}}{\sigma \sqrt{2}}} e^{-w^{2}} d w=\frac{1}{\sqrt{\pi}}\left[\int_{-\infty}^{0} e^{-w^{2}} d w+\int_{0}^{\frac{x-x_{w}}{\sigma \sqrt{2}}} e^{-w^{2}} d w\right]
$$

and

$$
M(x)=\frac{1}{\sqrt{\pi}}\left[\int_{0}^{-\infty} e^{-w^{2}} d w+\int_{0}^{\frac{x-x_{e}}{\sigma \sqrt{2}}} e^{-w^{2}} d w\right]
$$

or

$$
M(x)=\frac{1}{2}+\frac{1}{2} \operatorname{erf}\left(\frac{x-x_{\text {av }}}{\sigma \sqrt{2}}\right)
$$

or

$$
x=x_{\mathrm{av}}+\sigma \sqrt{2} \operatorname{erf}^{-1}[2 M(x)-1]
$$

which is just what we had before when $x<x_{a v}$. The distances proportional to erf ${ }^{-1}$ [2 $M(x)-1]$ will be 0 for $M(x)=1 / 2$ (or $x=x_{a v}$ ) and $+\infty$ when $M(x)=1$. The line will have a slope of $\sigma \sqrt{2}$ and an intercept of $x_{a v}$. Now the slope itself is awkward to determine since the scaling of the paper would have to be known. To avoid this difficulty, the value of $\sigma$ is estimated by considering the line at the points $x=x_{a v} \pm \sigma$. Then

$$
x_{a v} \pm \sigma=x_{a v}+\sigma \sqrt{2} \operatorname{erf}^{-1}\left[2 \mathrm{M}\left(x_{\mathrm{av}} \pm \sigma\right)-1\right]
$$


or

$$
\pm \frac{1}{\sqrt{2}}=\operatorname{erf}^{-1}\left[2 \mathrm{M}\left(\mathrm{x}_{\mathrm{av}} \pm \sigma\right)-1\right]
$$

and

$$
M\left(x_{a v} \pm \sigma\right)=\frac{1+\operatorname{erf}\left( \pm \frac{1}{\sqrt{2}}\right)}{2}
$$

The value of $\operatorname{erf}\left( \pm 1 / \gamma^{\prime} 2\right)$ is about \pm 0.68269 , so that $M\left(x_{a v}+\sigma\right)=0.8413$ and $\mathrm{M}\left(\mathrm{x}_{\mathrm{av}}-\sigma\right)=0.15865$. This, then, is the reason for the rule for obtaining the standard deviation of particle sizes by checking off the values of $x$ at the $84 \%$ and $16 \%$ cumulative mass fractions. 


\section{THE LOG-NOKMAL DISTRIBUTION AND THE VARIOUS AEROSOL DIAMETERS}

If the normal distribution were used to correlate particle sizes. an inherent difficulty would be encountered, for the size distribution is unsymmetrical. Particle sizes can be arbitrarily large, but they can never be less than zero. If, instead of using the particle radius, we use the logarithm of the radius, particle sizes ranging from zero to "infinity" are transformed to logarithms which range from $-\infty$ to $+\infty$. What advantage is obtained by this is significantly offset by the masking of the variability that is incurred. The percentage change in a quantity that increases from 100 to 200 is $[(200-100) / 100] \times 100 \%$ or $100 \%$. The percentage change in the natural logarithms is however only $15 \%$. The problem of testing whether log-normal correlations are in fact valid is a large and complex matter. We shall not go into it further here beyond observing that it is possible to get what appears to be correlations on matters that probably have nothing to do with log-normality.

We turn now to the various average radii discussed in connection with the lognormal distribution, such as those listed in Ref. 3. (We use radii here rather than diameters to avoid the typographical confusion with the " $d$ " of the differential. The conversion from radius to diameter in the formulas will be an obvious matter. We also adopt the notations $\mathrm{r}_{\mathrm{g}}$ and $\sigma_{\mathrm{g}}$ of Ref. 3 to facilitate comparisons.) The first thing to note is the fact that the moments of a log-normal distribution are themselves in a log-normal distribution. That is, if we say that the cumulative fraction of particles that have logarithms from $-\infty$ to $\ln r_{1}$ is in a log-normal distribution:

$$
N\left[\ln r_{1}\right]=\frac{1}{\ln \sigma_{B} \sqrt{2 \pi}} \int_{-\infty}^{\ln r_{1}} e^{-\frac{\left(\ln r-\ln r_{B}\right)^{2}}{2 \ln ^{2} \sigma_{8}}} d(\ln r)
$$

then functions of the form

$$
\frac{1}{\ln \sigma_{8} \sqrt{2 \pi}} \int_{-\infty}^{\ln \mathrm{r}_{1}} \mathrm{r}^{\mathrm{k}} \mathrm{e}^{-\frac{\left(\ln \mathrm{r}-\ln \mathrm{r}_{8}\right)^{2}}{2 \ln ^{2} \sigma_{8}}} \mathrm{~d}(\ln \mathrm{r})
$$

which we call the muments are also in a log-normal distribution. This, as will be seen, leads to great simplification in evaluating integrals like those of Eq. (26).

Equation (25) is seen to be the same formula as Eq. (16) for the ordinary normal distribution. Also, the constants $C_{1}$ and $C_{2}$ of Eq. (1) have been identified as $\ln \left(r_{g}\right)$ and 
$2 \ln ^{2}\left(\sigma_{g}\right)$, respectively. The quantity $r_{g}$ is called the geometric mean radius. The geometric mean $a_{g}$ of a number, $n$, of quantities $a_{i}$ is defined by the relation:

$$
a_{B}=\left(a_{1} a_{2} \ldots a_{n}\right)^{\frac{1}{n}}
$$

Taking the logarithm of both sides gives

$$
\ln \left(a_{8}\right)=\frac{1}{n} \sum_{i=1}^{n} \ln \left(a_{i}\right) .
$$

Thus, just as the quantity $\mu$ of Eq. (2) originates out of the idea of an arithmetic mean of a finite number of quantities, $\ln r_{g}$ is related to the arithmetic mean of the logarithms of a number of quantities. Furthermore, $\ln \sigma_{\mathrm{g}}$ now takes the role of the standard deviation of values of $\ln r$ about the mean value $\ln \mathrm{r}_{\mathrm{g}}$.

Returning to Eq. (26), we note that

$$
\left.\frac{1}{\sqrt{2 \pi} \ln \sigma_{8}} \int_{-\infty}^{\ln \left(x_{1}\right)} \mathrm{r}^{k} \mathrm{e}^{-\frac{\left(\ln r-\ln r_{8}\right)^{2}}{2 \ln ^{2} \sigma_{8}}} \mathrm{~d}(\ln \mathrm{r})=\frac{1}{\sqrt{2 \pi} \ln \sigma_{B}} \int_{-\ldots}^{\ln \left(x_{1}\right)} \mathrm{e}^{-\left[\frac{\left(\ln r-\ln r_{8}\right)^{2}}{2 \ln ^{2} \sigma_{2}}-\mathrm{k} \ln r\right.}\right] \mathrm{d}(\ln \mathrm{r})
$$

since $\mathrm{r}^{\mathrm{k}}=\mathrm{e}^{\mathrm{k} \ln (\mathrm{r})}$.

Now the exponent can be rearranged as follows:

$$
\frac{\left(\ln r-\ln r_{B}\right)^{2}}{2 \ln ^{2} \sigma_{8}}-k \ln r=\frac{\ln ^{2} r-2 \ln r\left(\ln r_{g}+k \ln ^{2} \sigma_{8}\right)+\ln ^{2} r_{g}}{2 \ln ^{2} \sigma_{B}}
$$

This, by the "completing the square" operation of elementary algebra, is:

$$
\frac{\left[\ln r_{8}-\left(\ln r_{8}+k \ln ^{2} \sigma_{B}\right)\right]^{2}}{2 \ln ^{2} \sigma_{z}}-\left(\frac{k^{2}}{2} \ln ^{2} \sigma_{8}+k \ln r_{g}\right) \text {. }
$$


Our distribution in Eq. (29) is then transformed thus:

$$
\frac{1}{\sqrt{2 \pi} \ln \sigma_{8}} \int_{-\infty}^{\ln r_{1}} r^{k} e^{-\frac{\left(\ln r-\ln r_{8}\right)^{2}}{2 \ln ^{2} \sigma_{8}}} d(\ln r)=\frac{e^{\frac{k^{2}}{2} \ln ^{2} \sigma_{8}+k \ln r_{8}}}{\sqrt{2 \pi} \ln \sigma_{8}} \int_{-\infty}^{\ln r_{1}} e^{-\frac{\left(\ln r-\left.\left(\ln r_{8}+k \ln ^{2} \sigma_{8}\right)\right|^{2}\right.}{2 \ln ^{2} r_{8}}} d(\ln r) .
$$

This is still a log-normal distribution with the same standard deviation term but with a "displaced" value of the mean value of $\ln (r)$, denoted by $\ln \overline{r_{k}}$, where $\overline{r_{k}}$ is a new geometric mean radius

$$
\ln \overline{\mathrm{r}}_{\mathrm{k}}=\ln \mathrm{r}_{\mathrm{g}}+\mathrm{k} \ln ^{2} \sigma_{\mathrm{g}} \text {. }
$$

Letting $\ln \left(\mathrm{r}_{1}\right)$ go to $+\infty$, Eq. (32) becomes:

$$
\frac{1}{\sqrt{2 \pi} \ln \sigma_{g}} \int_{-\infty}^{\infty} r^{k} e^{-\frac{\left(\ln r-\ln r_{B}\right)^{2}}{2 \ln ^{2} \sigma_{g}}} d(\ln r)=\frac{e^{\frac{k^{2}}{2} \ln ^{2} \sigma_{B}+k \ln r_{B}}}{\sqrt{2 \pi} \ln \sigma_{B}} \int_{-\infty}^{\infty} e^{-\frac{\left(\ln r-\ln \overline{r_{k}}\right)^{2}}{2 \ln ^{2} \sigma_{B}}} d(\ln r)
$$

and, just as was done for Eq. (2) we make the substitution

$$
w=\frac{\ln r-\ln \overline{r_{k}}}{\sqrt{2} \ln \sigma_{8}}
$$

The right-hand side of Eq. (34) is then

$$
\frac{e^{\frac{k^{2}}{2} \ln ^{2} \sigma_{a}+k \ln r_{2}}}{\sqrt{\pi}} \int_{-\infty}^{\infty} e^{-w^{2}} d w
$$

Since Eq. (5)

$$
\int_{-\infty}^{\infty} e^{-w^{2}} d w=\sqrt{\pi}
$$


we have finally:

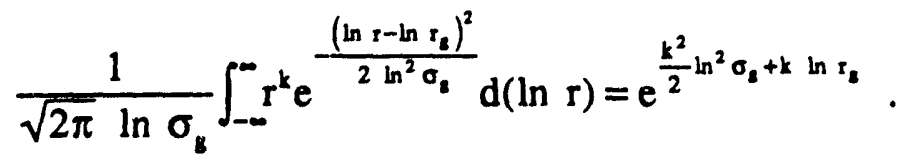

We can now readily generate all of the radii (diameters) of p. 113 of Ref. (1):

1. "The radius (diameter) of the particle $r_{m}\left(d_{m}\right)$ having a volume equal to the average volume or mass."

$$
\left(r^{3}\right)_{a v}=\frac{\frac{1}{\sqrt{2 \pi\left(\ln \sigma_{g}\right)}} \int_{-\infty}^{\infty} r^{3} e^{-\frac{\left(\ln r-\ln r_{g}\right)^{2}}{2 \ln ^{2} \sigma_{g}}} d(\ln r)}{\frac{1}{\sqrt{2 \pi} \ln \sigma_{g}} \int_{-\infty}^{-\infty} e^{-\frac{\left(\ln r-\ln r_{g}\right)^{2}}{2 \ln ^{2} \sigma_{g}}} d(\ln r)}
$$

and the average volume is $(4 / 3) \pi\left(r^{3}\right)$ av. Multiplying by a particle density gives an average mass. As was shown in the discussion of Eqs. (2)-(5), the denominator is 1 , and the numerator is obtained by Eq. (35):

$$
\left(r^{3}\right)_{a v}=e^{4.5 \ln ^{2} \sigma_{8}+3 \ln \mathrm{r}_{8}}
$$

We define $r_{m}$ as $r_{m}^{3} \equiv\left(r^{3}\right)_{a v}$ so that $\ln r_{m^{3}}=\ln \left(r^{3}\right)_{a v}=4.5 \ln 2 \sigma_{g}+3 \ln r_{g}$ and finally

$$
\ln r_{m}=1.5 \ln ^{2} \sigma_{g}+\ln r_{g}
$$

(We note that since $r_{m}=0.5 d_{m}, r_{g}=0.5 d_{g}$, then $\ln \left(d_{m}\right)=1.5 \ln ^{2} \sigma_{g}+\ln d_{g}$ )

2. "The radius (diameter) of the particle, $r_{a}$, having a surface area equal to the average surface area."

$$
\begin{aligned}
\left(r^{2}\right)_{a v} & =\frac{1}{\sqrt{2 \pi} \ln \sigma_{8}} \int_{-\infty}^{-} r^{2} e^{-\frac{\left(\ln r-\ln r_{8}\right)^{2}}{2 \ln ^{2} \sigma_{8}}} d(\ln r) \\
& =e^{2 \ln ^{2} \sigma_{8}+2 \ln r_{8}}
\end{aligned}
$$

by Eq. (35). 
Here, $r_{a}$ is defined by the relation

$$
\mathrm{ra}^{2} \equiv\left(\mathrm{r}^{2}\right) \mathrm{av}
$$

so that

$$
\ln \mathrm{r}_{\mathrm{a}}=1.0 \ln ^{2} \sigma_{\mathrm{g}}+\ln \mathrm{r}_{\mathrm{g}}
$$

$$
\text { and } \ln d_{a}=1.0 \ln 2 \sigma g+\ln d g .
$$

3. "The mean radius, $\left(r_{m}\right)_{a v}$, of the mass or volume distribution."

$$
\left(\overline{r_{m}}\right)=\frac{\frac{1}{\sqrt{2 \pi} \ln \sigma_{g}} \int_{-\infty}^{-\infty} r \cdot r^{3} e^{-\frac{\left(\ln r-\ln r_{g}\right)^{2}}{2 \ln ^{2} \sigma_{g}}} d(\ln r)}{\frac{1}{\sqrt{2 \pi} \ln \sigma_{g}} \int_{-\infty}^{\infty} r^{3} e^{-\frac{\left(\ln r-\ln r_{g}\right)^{2}}{2 \ln ^{2} \sigma_{g}}} d(\ln r)} .
$$

Applying Eq. (35), we can obtain

$$
\left(\overline{r_{m}}\right)=\frac{\left[\left(k+\frac{1}{2}\right) \ln { }^{2} \sigma_{8}+\ln r_{8}\right]}{e^{\left[\frac{k^{2}}{2} \ln ^{2} \sigma_{8}+k \ln r_{8}\right]}}=e
$$

and finally, with $k=3$

$$
\begin{aligned}
& \left(\overline{r_{m}}\right)=e^{3.5 \ln ^{2} \sigma_{\mathrm{g}}+\ln r_{\mathrm{g}}} \\
& \text { and } \ln \left(r_{m}\right)=3.5 \ln ^{2} \sigma_{\mathrm{g}}+\ln \mathrm{r}_{\mathrm{B}} \\
& \text { or } \ln d_{m}=3.5 \ln ^{2} \sigma g+\operatorname{lndg} .
\end{aligned}
$$

4. "The mean radius, $\left(\bar{r}_{a}\right)$, of the area distribution."

For this, we can use Eq. (41), but with $k=2$ instead of 3:

$$
\left(\bar{r}_{1}\right)=\frac{e^{4.5 \ln ^{2} \sigma_{8}+3 \ln r_{4}}}{e^{2 \ln ^{2} \sigma_{8}+2 \ln r_{8}}}
$$


and

$$
\begin{aligned}
& \ln \left(\bar{r}_{\mathrm{B}}\right)=2.5 \ln ^{2} \sigma_{\mathrm{B}}+\ln \mathrm{r}_{\mathrm{B}} \\
& \text { or } \ln \overline{\mathrm{d}}_{\mathrm{z}}=2.5 \ln ^{2} \sigma_{\mathrm{B}}+\ln \mathrm{dg} .
\end{aligned}
$$

5. "The mass median radius, $r^{\prime} \mathrm{m}$ "

This is the "geometric mean radius" for the volume or mass distribution, obtainable immediately from Eq. (33):

$$
\begin{aligned}
& \ln r_{m}^{\prime}=\ln r_{g}+3 \ln ^{2} \sigma_{g} \\
& \ln d_{m}=\ln d g+3.0 \ln ^{2} \sigma_{g} .
\end{aligned}
$$

6. "The area median radius, $\mathrm{r}_{\mathrm{a}}$."

This is the "geometric mean radius" for the area distribution, $k=2$ in Eq. (33):

$$
\begin{gathered}
\ln r_{a}^{\prime}=\ln r_{g}+2 \ln ^{2} \sigma_{g} \\
\ln d_{a}^{\prime}=\ln d g+2.0 \ln ^{2} \sigma_{g} .
\end{gathered}
$$

Finally, there is the quantity $\mathrm{d}^{*}$, called the "diameter of the count mode." This is the diameter itself, not the logarithm of the diameter, for which the count rate reaches a peak. The frequency distribution for $\ln (r)$, the number of particles for which the logarithm of the radius lies between $\ln (r)$ and $\ln (r)+d[\ln (r)]$ is [see Eq. (25)]:

$$
\frac{1}{\sqrt{2 \pi} \ln \sigma_{g}} e^{\frac{\left(\ln r-\ln r_{g}\right)^{2}}{2 \ln ^{2} \sigma_{g}}} d(\ln r)
$$

This has its maximum at $\ln (r)=\ln \left(r_{g}\right)$. The frequency distribution for the radius itself, however, is:

$$
\frac{1}{\sqrt{2 \pi} \ln \sigma_{8}} e^{-\frac{\left(\ln r-\ln r_{8}\right)^{2}}{2 \ln ^{2} \sigma_{8}}} \frac{d r}{r}
$$


This has its maximum, as Fig. 5.14 of Ref. (1) shows, at a different place, $r^{*}$, which is obtainable by finding the maximum of

$$
y(r)=\frac{1}{r} e^{-\frac{\left(\ln r-\ln r_{g}\right)^{2}}{2 \ln ^{2} \sigma_{z}}}
$$

Differentiating with respect to $r$ and setting the right-hand side equal to zero gives

$$
\ln \left(\mathrm{r}^{*}\right)=\ln \left(\mathrm{r}_{\mathrm{g}}\right)-\ln ^{2}\left(\sigma_{\mathrm{g}}\right)
$$




\section{SUMMARY}

In this paper, the connection between the log-normal distribution and the normal distribution is shown; the mathematical basis for the various diameters used in the aerosol literature was developed, and the reasoning behind the graphical presentation on so-called "log-normal" graph paper has been presented. While none of this discussion is an original development, the purpose has been to clarify the often confusing terminology and to gather together in one place a consistent, unambiguous explanation of these matters. 


\section{REFERENCES}

1. R. Dennis, Editor Handbook on Aerosols, TID-26608, Technical Information Center, U.S. Department of Energy (1976).

2. S. M. Berman, Mathematical Statistics, Intext Educational Publishers (1971).

3. B. O. Peirce, A Short Table of Integrals, 3rd Edition, Ginn and Company (1929), p. 63, Formula 492 .

4. T. Hatch, and S. P. Choate, "Statistical Description of the Size Properties of NonUniform Particulate Substances," J. Franklin Inst., 207, 369 (1929), cited in Ref. 6.

5. M. Abramowitz and I. A. Stegun, Editors, Handbook of Mathematical Functions, National Bureau of Standards Applied Mathematics Series No. 55, U.S. Government Printing Office, Seventh Printing, (1968).

6. W. C. Hinds, Aerosol Technology, John Wiley \& Sons (1982).

7. J. Aitchison and J.A.C. Brown, The Lognormal Distribution, Cambridge University Press (1969). 


\section{INTERNAL DISTRIBUTION}

1. S. Bloom

2. M. S. Childers

3. L. Dresner

4. S. E. Fisher

5. D. T. James

6. J. E. Jones Jr.

7. R. A. Just

8. T. S. Kress

9. K. E. Lenox

10. T. McSweeney, Battelle

11. S. C. Nelson

12. J. C. Pease

13-17. M. L. Tobias

18. M. B. Townsend
19. R. Wall

20. R. P. Wichner

21. V. K. Wilkinson

22. B. K. Williams

23. S. R. Wilson

24. A. L. Wright

25. J. M. Young

26. A. K. Zava

27. ORNL Patent Section

28. Central Research Library

29. Document Reference Section

30-31. Laboratory Records Department

32. Laboratory Records (RC)

\section{EXTERNAL DISTRIBUTION}

33. Office of Assistant Manager for Energy Research and Development, DOE-ORO, Oak Ridge, TN 37831

34-35. Office of Scientific and Technical Information, P.O. Box 62, Oak Ridge, TN 37831 

